Harold A. McAlister and William I. Hartkopf (eds.)

\title{
Detection and Measurement of Binary Stars with the HST Fine Guidance Sensors
}

\author{
O. G. FRANZ, T. J. KREIDL, \& L. H. WASSERMAN \\ Lowell Observatory, 1400 W. Mars Hill Rd., Flagstaff, AZ 86001, USA
}

\author{
A. BRADLEY \\ Allied Signal Aerospace Co., P.O. Box 91, Annapolis Jct., MD 20701, USA \\ E. NELAN, D. STORY, \& A. L. WHIPPLE \\ University of Texas, Department of Astronomy, Austin, TX 78712, USA
}

\begin{abstract}
After briefly describing the operation of the Hubble Space Telescope Fine Guidance Sensors (HST-FGS) in the Transfer Function (TF) Scan mode, we discuss the reduction and analysis of scan data affected by HST jitter and Optical Field Angle Distortion (OFAD). We present relative positions and magnitude differences for the components of ADS $11300=\mathrm{Hu} 581=$ WDS $18229+1458$ derived from TF scans obtained on 17 February 1992. Residuals from a newly revised orbit provide a first indication of the accuracy of HST-FGS observations in the Transfer Function Scan mode.
\end{abstract}

\section{INTRODUCTION}

In the two years elapsed since the launch of Hubble Space Telescope, only two astrometric projects have been carried out with the HST Fine Guidance Sensors (FGS) in the Transfer Function (TF) Scan mode: A study of ADS $11300=\mathrm{Hu}$ 581 = WDS 18229+1458 (Franz et al. 1991) and a search for binaries among bright members of the Hyades cluster (Franz et al. 1992). This experience, although limited, demonstrates great potential for binary-star research with the HST-FGS in TF-scan mode.

\section{OBSERVATIONS}

The Fine Guidance Sensors are two-axis interferometers, each based upon two orthogonal Koester prisms and using two pairs of photomultiplier tubes (PMTs). Observation in the TF-scan mode takes place as follows:

1. The Instantaneous Field Of View (IFOV) is positioned by the flight computer to the expected target location.

2. The flight computer commands the FGS to initiate Search and Track mode. The IFOV moves outward in a spiral motion until the total counts from the four PMTs exceed a predefined threshold, indicating star presence. Following successful Search mode, Coarse Track mode is commanded. In Coarse Track the FGS IFOV will nutate about the star position at a rate of once per second and with a nutation radius of $2^{\prime \prime} .5$. The objective of Coarse Track is to balance out the counts in each PMT, thereby defining the target position at the center of nutation. 
3. The flight computer then commands the FGS to move to the start-scan position at a preset distance along a $45^{\circ}$ angle from the center of nutation.

4. The flight computer transmits Star Selector Servo rate commands to the FGS which move the IFOV across the target at a constant rate, in alternate directions, for a preset number of times and duration. The minimum commandable scan rate is $0^{\prime \prime} .001 \mathrm{sec}^{-1}$. A full discussion of the flight hardware and of the ground system for HST astrometry has been presented by Bradley et al. (1991).

Telemetry of the sampled PMT outputs (A,B) and of the corresponding Star Selector Servo angles provides the raw data to generate, for each interferometer axis $(\mathrm{X}, \mathrm{Y})$, a step-by-step Transfer Function (TF), often referred to as S-curve, of the general form $S=(A-B) /(A+B)$.

ADS 11300 was first observed with HST-FGS on 1 November 1990. A follow-up series of TF-scans began with the observation of 17 February 1992. The scan-rate for this observation was $0 " 04 \mathrm{sec}^{-1}$ along each interferometer axis. A single-scan duration of 30 sec and a PMT sampling rate of $40 \mathrm{~Hz}$ thus yielded, along each axis, 1200 steps of $0^{\prime \prime} .001$ each. Both observations were carried out with FGS3. The spacecraft was under Fine Lock guiding, and realtime transmission of data was in effect.

\section{DATA REDUCTION AND ANALYSIS}

Determination of relative component positions and magnitude differences for double stars scanned with HST-FGS is based on the assumption that the TF of a double star is a linear superposition of two single-star functions (Franz et al. 1991). Simple in principle, the analysis is complicated by the effects of spacecraft jitter (boresight motion) upon TF scans. This jitter is dominated by thermally-induced solar-array vibrations in excess of pre-launch estimates.

An effort to deal directly with jitter has led to changes of the on-board spacecraft control law. These modifications, denoted as Solar Array Gain Augmentation (SAGA), represent a filter incorporated into the vehicle control law to attenuate or dampen the spacecraft motions due to Solar Array disturbances. The SAGA filter in use during the past year was designed to attenuate a $0.1 \mathrm{~Hz}$ vibration mode in the jitter spectrum. Reduction of a $0.6 \mathrm{~Hz}$ vibration, presently the dominant mode, is the objective of a modification to the SAGA filter now undergoing tests. Although the use of SAGA during the past year has improved the quality of TF-scans, effects of jitter remain. Data reduction must deal with these effects.

Bradley (1990) has devised a rather general and effective method of jitter removal from TF-scan data. His algorithm applies the position errors sensed by the dominant guiding FGS to the astrometry FGS, thereby largely removing the effects of jitter from TF-scan data as illustrated in Figure 1. There we have plotted, from a series of ten single-star calibration TFs obtained on 13 February 1992, one seemingly undisturbed and one noticeably jitter-corrupted $\mathrm{Y}$-scan before and after jitter removal. The efficacy of the algorithm is readily apparent. 


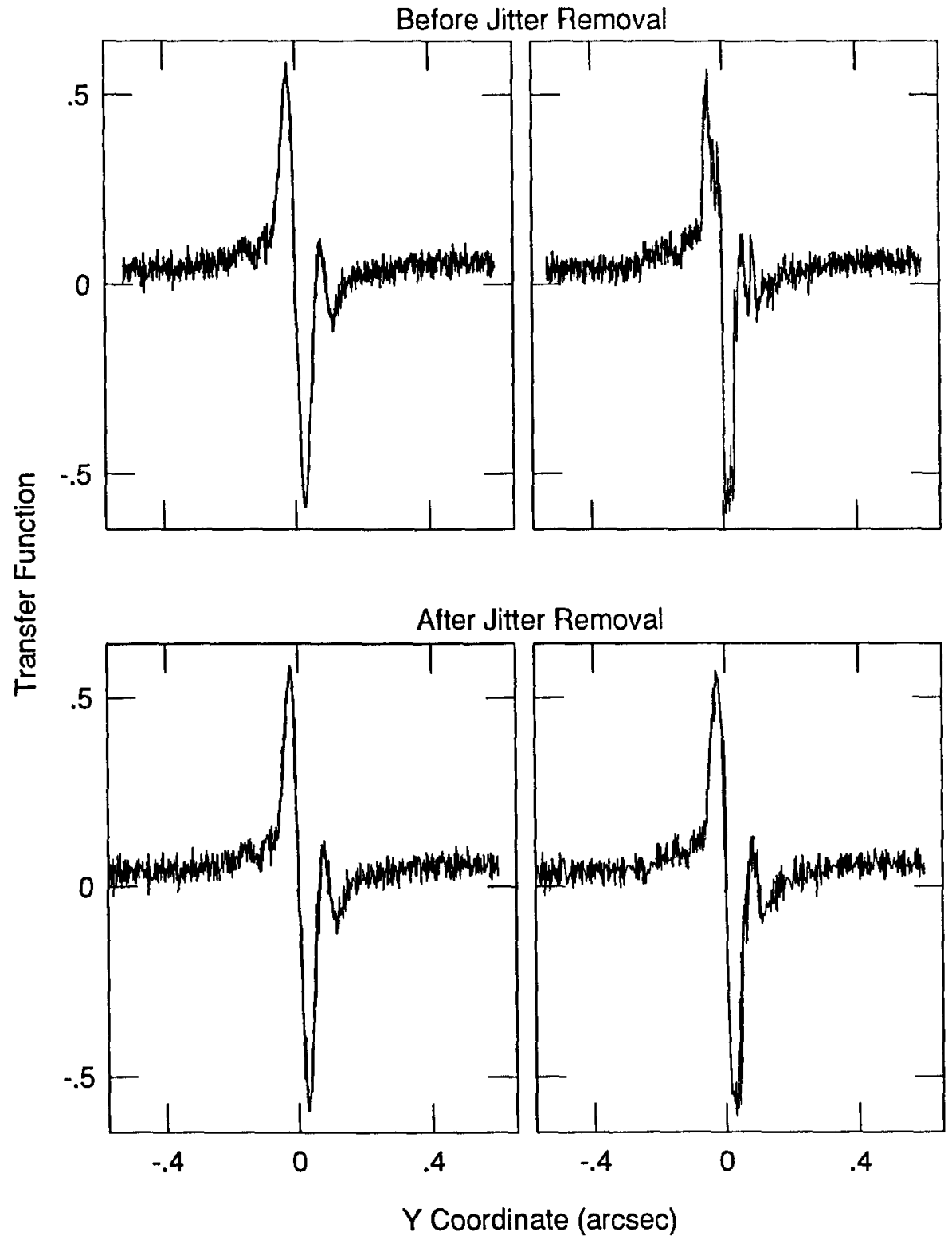

FIGURE 1. A seemingly undisturbed (left) and a noticeably jitter-corrupted (right) Transfer Function Y-scan of a single star before (top) and after (bottom) jitter removal. 

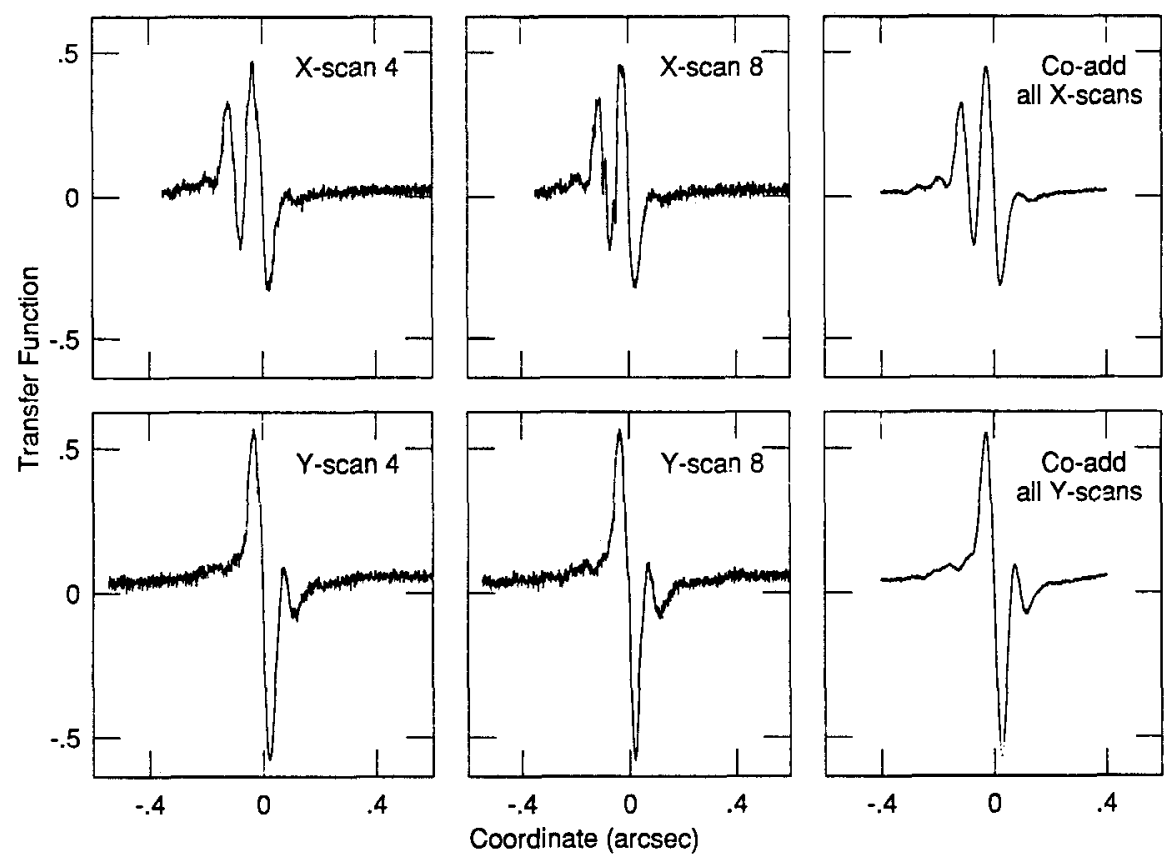

FIGURE 2. Best (left) and worst (middle) of ten Transfer Function scans in FGS $X$ (top) and $Y$ (bottom) of ADS 11300 obtained with FGS3 on 17 February 1992. Also shown (right) is the result of co-addition of all ten scans after jitter removal.

The best and the worst of ten TF-scans of ADS 11300 in FGS X and Y coordinates obtained on 17 February 1992 are shown in Figure 2, together with the co-addition of all ten scans after jitter removal. The $\mathrm{X}$-axis TFs clearly indicate a well separated pair. The $\mathrm{Y}$-axis TFs, on the other hand, cannot be distinguished from those of a single star. By coincidence, the spacecraft roll angle at the time of observation was such that the FGS X-axis was oriented at the position angle of the pair. Analysis of the TFs yields the magnitude difference of the components of ADS 11300 and relative positions in the FGS $\mathrm{X}-\mathrm{Y}$ frame.

Jitter removal is not merely a matter of cosmetics or aesthestics. In Figure 3 we show as open circles the separations of ADS 11300 on the FGS X-axis derived from the ten individual scans before jitter removal. These projected separations vary from less than $0^{\prime \prime} .087$ to more than $0^{\prime \prime} .093$. Analysis of the same scans after jitter removal yields the separations plotted in Figure 3 as filled circles, showing a peak-to-peak scatter of less than 2 mas. Note, also, that the average separation from corrected scans is 2 mas larger than that derived from uncorrected TFs. The separations in FGS X, rounded to $0 " .001$, together with the derived magnitude differences, are given in Table 1. Also listed are their means and the values derived from TFs generated by co-addition of all scans before and after jitter removal. Since on the FGS Y-axis the pair could not be resolved, we adopted $0^{\prime \prime} .000$ as the separation in $Y$.

Before transforming the component-positions from the FGS3 X-Y frame to 


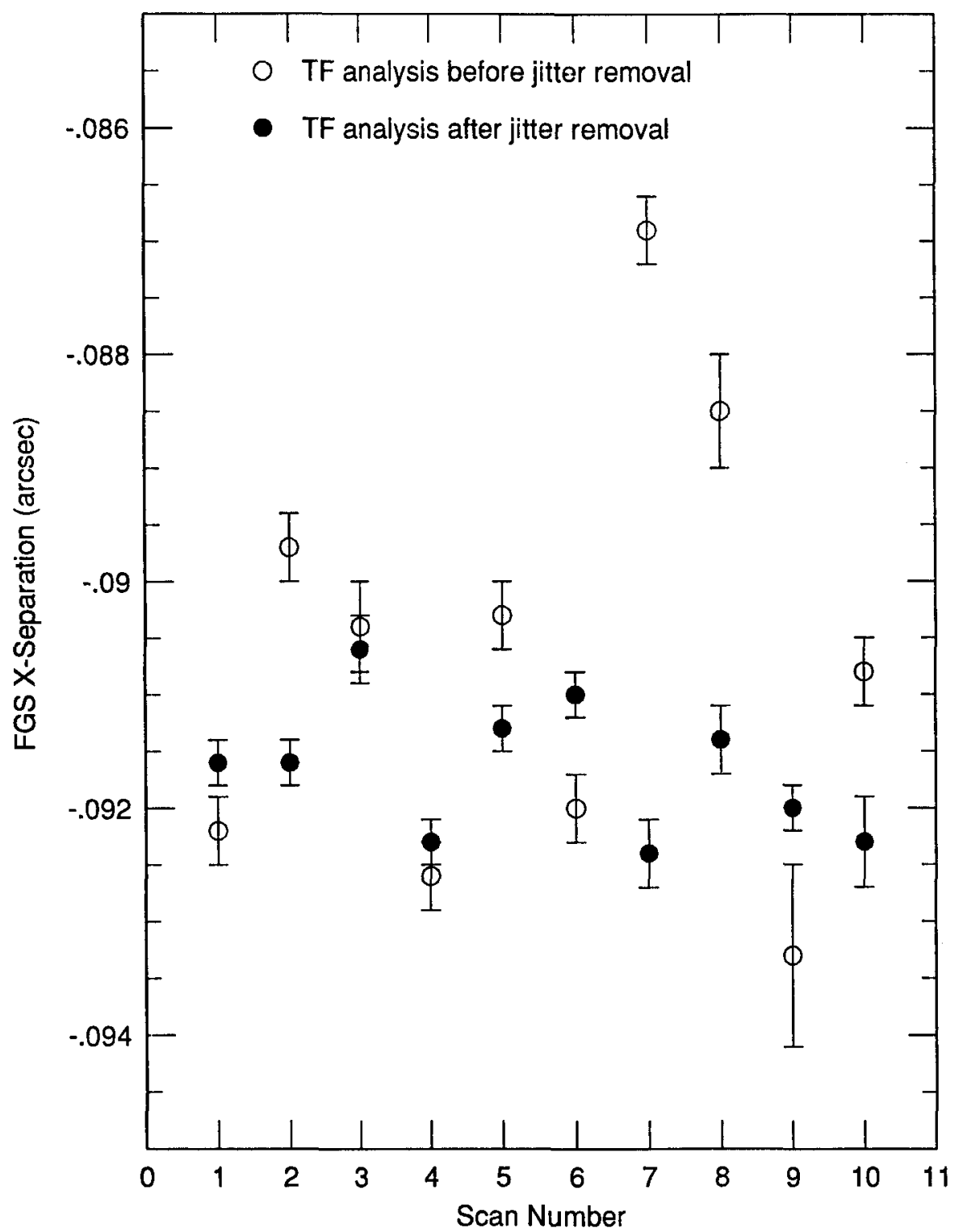

FIGURE 3. Angular separations of ADS 11300 in the FGS X coordinate derived from ten Transfer Function scans before (open circles) and after (filled circles) jitter removal. 
TABLE 1. Results from 10 Scans of ADS 11300 on the FGS X-axis

\begin{tabular}{|c|c|c|c|c|}
\hline & \multicolumn{2}{|c|}{$\begin{array}{c}\text { After } \\
\text { Jitter Removal }\end{array}$} & \multicolumn{2}{|c|}{$\begin{array}{c}\text { Before } \\
\text { Jitter Removal }\end{array}$} \\
\hline & Sep. & mag. diff. & Sep. & mag. diff. \\
\hline & $\begin{array}{c}0^{\prime \prime} .092 \\
0.092 \\
0.091 \\
0.092 \\
0.091 \\
0.091 \\
0.092 \\
0.091 \\
0.092 \\
0.092\end{array}$ & $\begin{array}{l}0.68 \\
0.71 \\
0.68 \\
0.75 \\
0.67 \\
0.72 \\
0.70 \\
0.68 \\
0.67 \\
0.65\end{array}$ & $\begin{array}{r}0.092 \\
0.090 \\
0.090 \\
0.093 \\
0.090 \\
0.092 \\
0.087 \\
0.089 \\
0.093 \\
0.091\end{array}$ & $\begin{array}{l}0.63 \\
0.70 \\
0.63 \\
0.75 \\
0.72 \\
0.62 \\
0.71 \\
0.66 \\
0.68 \\
0.71\end{array}$ \\
\hline $\begin{array}{c}\text { mean } \\
\text { co-added }\end{array}$ & $\begin{array}{r}0 " .092 \\
0.092\end{array}$ & $\begin{array}{l}0.69 \\
0.69\end{array}$ & $\begin{array}{r}0.090 \\
0.090\end{array}$ & $\begin{array}{l}0.68 \\
0.67\end{array}$ \\
\hline
\end{tabular}

the equatorial system, we also corrected them for the nominal effects of OFAD. These effects are inherent in the optical design of the FGS and are not a consequence of the spherical aberration in the HST primary mirror. Final values of the relative positions from both observations of ADS 11300 are listed in Table 2.

TABLE 2. ADS 11300: Relative Positions for Equinox 2000 from HST-FGS Observations

\begin{tabular}{|ccccc|}
\hline$t$ & $x$ & $y$ & $\theta$ & $\rho$ \\
\hline 1990.836 & $0^{\prime \prime} .018$ & $-0^{\prime \prime} .062$ & $285^{\circ} .75$ & $0^{\prime \prime} .065$ \\
1992.132 & $0^{\prime \prime} .032$ & $-0^{\prime \prime .084}$ & $290^{\circ} .66$ & $0^{\prime \prime} .089$ \\
\hline
\end{tabular}

\section{DISCUSSION}

ADS 11300 was selected in 1990 for observation under the HST Early Release Observation (ERO) project. Its selection was prompted by a preliminary orbit by Baize (1974) which suggested that the pair, after recent periastron passage, may have a current angular separation well below 0 ".1. A revised orbit based on all visual measures through 1977 and on the HST-FGS observation of 1990 indicated a time of periastron $\mathrm{T}=1992.1 \pm 0.6$, followed by a rapid decrease in separation to less than $0^{\prime \prime} .01$ by mid-1992. ADS 11300 thus appeared to provide an exceptional opportunity to test and calibrate the resolution and detection capability of HST-FGS in the TF-scan mode (Franz et al. 1991).

The observation of 1992.132 clearly did not meet this expectation. A newly revised orbit (Figure 4), incorporating both HST-FGS measures, indicates that periastron passage may not occur before 1994. The residuals from the new orbit, 


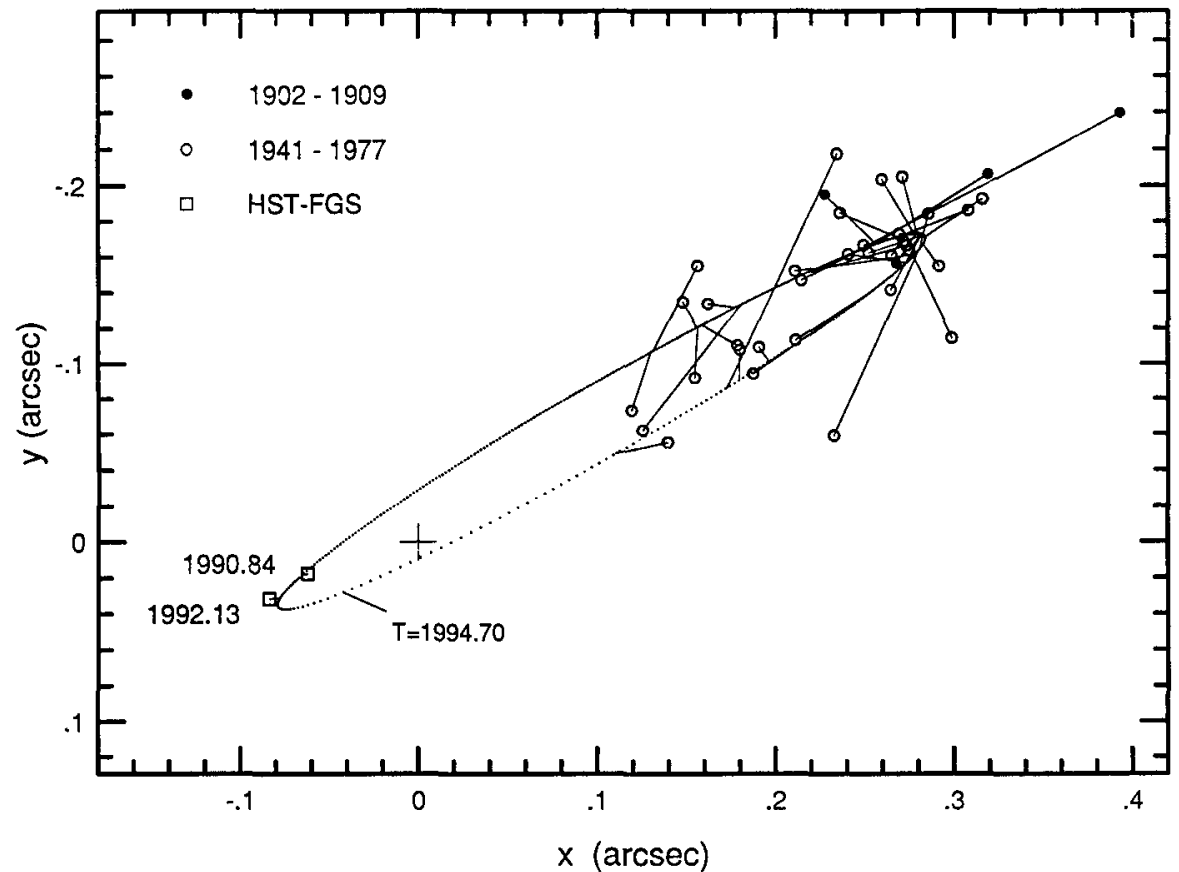

FIGURE 4. Revised orbit of ADS 11300 based upon 37 visual data points and two HST-FGS3 observations. T indicates the predicted time of periastron passage. A straight line connects each data point with its corresponding position on the apparent orbit.

however, provide the opportunity for a tentative assessment of the accuracy of these measures. In the orbit solution, we assigned unit weight to each visual measure. Because there are nearly 40 visual data points, the combined weight of the two HST observations should be at least 40. Assuming an accuracy at least equal to that of "miscellaneous" speckle measures usually given weight 5 , we adopted 100 as a reasonable weight for each HST-FGS observation. The residuals so derived are listed in Table 3.

TABLE 3. ADS 11300: Residuals of FGS Measures from Revised Orbit

\begin{tabular}{|cccc|}
\hline $\mathrm{t}$ & $\Delta \rho$ & $\Delta \theta$ & $\rho \times \Delta \theta$ \\
\hline 1990.836 & $-0^{\prime \prime} .004$ & $0^{\circ} .01$ & $0^{\prime \prime} 000$ \\
1992.132 & $0^{\prime \prime} .006$ & $-1^{\circ} .02$ & $-0^{\prime \prime} .001$ \\
\hline
\end{tabular}

If we view these residuals as indicators of the accuracy of HST-FGS astrometry in the TF-Scan mode, we must remember that both observations were made with an uncalibrated instrument, and that owing to the spacecraft orientation during the 1992.132 observation, the true separation along the FGS Y-axis remained unknown. Further observations of ADS 11300 now under way or being planned should soon show whether these residuals are representative of the performance of HST-FGS in the Transfer Function Scan mode. 


\section{CONCLUSIONS AND OUTLOOK}

We will shortly begin observations of binaries with well determined orbits based on extensive series of measures with the CHARA speckle camera. These observations should produce reliable data for calibrating the FGS in the TF-scan mode. They should also yield information on limits of angular resolution and binary detection. "Single" stars of early and late spectral type will be observed to examine the dependence of TFs on color temperature.

Programs approved though not yet scheduled include measurement of known binaries at critical orbital phases; examination of several late-type dwarf stars claimed to have low-mass "astrometric" companions that should be detectable by FGS scans; and a search for duplicity among faint and highly probable members of the Hyades cluster. We are optimistic, even confident, that HST-FGS in the Transfer Function Scan mode will prove to be a powerful and productive tool in binary star research.

\section{ACKNOWLEDGEMENTS}

We thank W. I. Hartkopf for making available his software for binary-star orbit computation and for helpful advice on its use. This work was carried out with support from the NASA Marshall Space Flight Center under contract NAS832906 and the Goddard Space Flight Center under contract NAS5-29285 and under grant NAG5-1603 to the University of Texas at Austin.

\section{REFERENCES}

Baize, P. 1974, $A \& A, 13,65$

Bradley, A. 1990 , private communication

Bradley, A., Abramowicz-Reed, L., Story, D., Benedict, G., \& Jefferys, W. 1991, PASP, 103,317

Franz, O.G., Kreidl, T.J.N., Wasserman, L.H., Bradley, A.J., Benedict, G.F., Hemenway, P.D., Jefferys, W.H., McArthur, B., McCartney, J.E., Nelan, E., Shelus, P.D., Story, D., Whipple, A.L., Duncombe, R.L., Fredrick, L.W., \& van Altena, W.F. $1991, A p J, 377$, L17

Franz, O.G., Wasserman, L.H., Nelan, E., Lattanzi, M.G., Bucciarelli, B., \& Taff, L.G. $1992, A J, 103,190$

\section{DISCUSSION}

MATHIEU: From a practical point of view, do GO's from the community have the technical proficiency to, first, write an FGS proposal and, second, reduce the resultant data?

FRANZ: You may find the proposal process more intimidating than the reduction of the data. We would certainly be happy to make software, developed at the Lowell Observatory, available and to provide advice and assistance. Also, there will be in place at STScI a pipe-line data reduction and analysis system. I suspect that Dr. Taff will address that point. 